
Not for reproduction, distribution or commercial use.

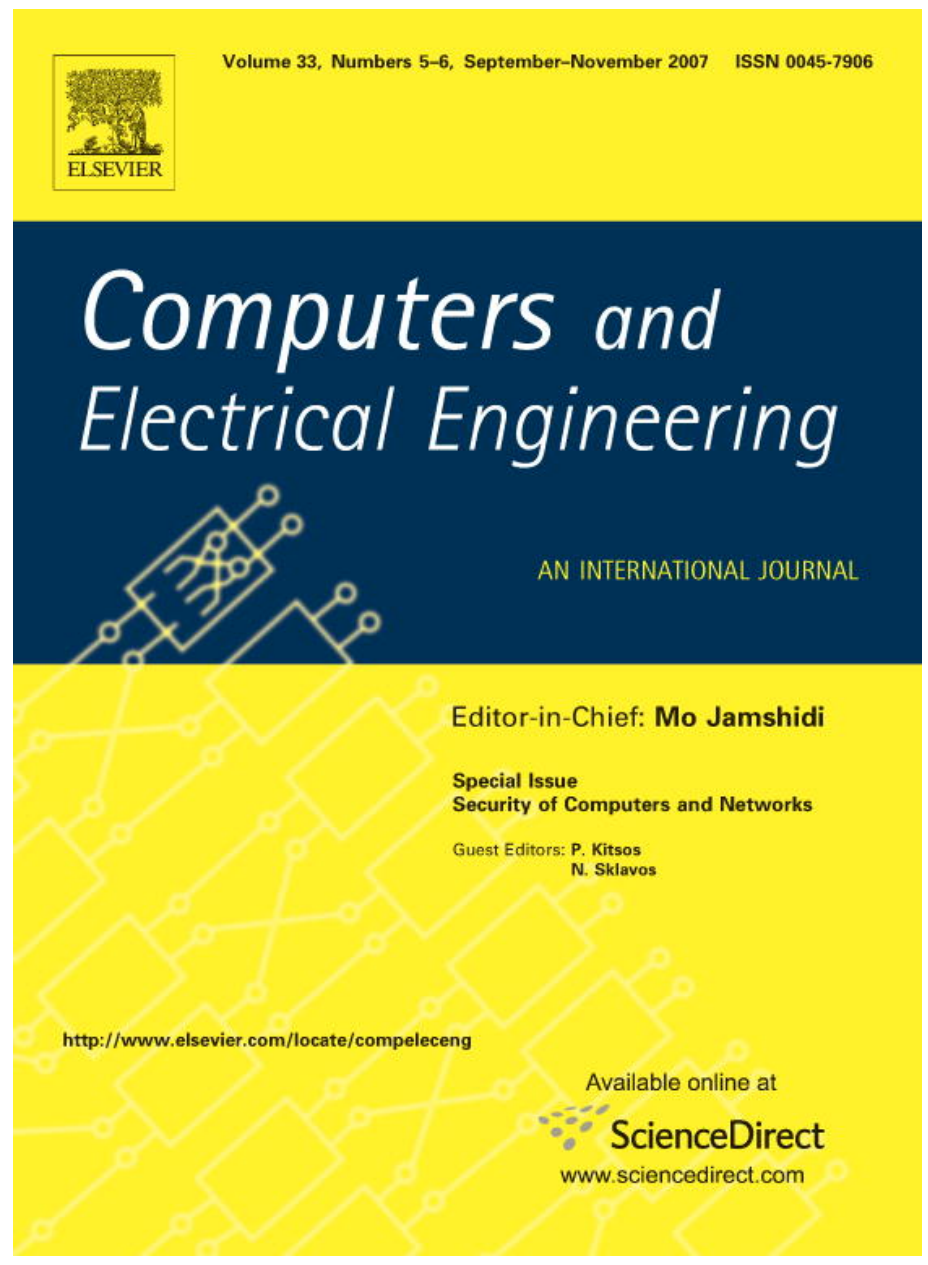

This article was published in an Elsevier journal. The attached copy

is furnished to the author for non-commercial research and education use, including for instruction at the author's institution, sharing with colleagues and providing to institution administration.

Other uses, including reproduction and distribution, or selling or licensing copies, or posting to personal, institutional or third party websites are prohibited.

In most cases authors are permitted to post their version of the article (e.g. in Word or Tex form) to their personal website or institutional repository. Authors requiring further information regarding Elsevier's archiving and manuscript policies are encouraged to visit: 


\title{
Differential power and electromagnetic attacks on a FPGA implementation of elliptic curve cryptosystems
}

\author{
E. De Mulder ${ }^{\text {a }}$, S.B. Örs ${ }^{\text {b,* }}$, B. Preneel ${ }^{\text {a }}$, I. Verbauwhede ${ }^{\text {a }}$ \\ ${ }^{\text {a }}$ Katholieke Universiteit Leuven, Department of Electrical Engineering, SCD/COSIC, Belgium \\ ${ }^{\mathrm{b}}$ Istanbul Technical University, Department of Electronics and Communication Engineering, Turkey
}

Available online 5 July 2007

\begin{abstract}
This paper describes the first differential power and electromagnetic analysis attacks performed on a hardware implementation of an elliptic curve cryptosystem. In the same time we also compared the metrics used in differential power and electromagnetic radiation attacks. We describe the use of the Pearson correlation coefficient, the distance of mean test and the maximum likelihood test. For each metric the number of measurements needed to get a clear idea of the right guess of the key-bit is taken as indication of the strength of the metric.
\end{abstract}

(C) 2007 Elsevier Ltd. All rights reserved.

Keywords: FPGA; Power analysis; Electromagnetic analysis; Elliptic curve cryptosystems

\section{Introduction}

Elliptic curve cryptography (ECC) was proposed independently by Miller [13] and Koblitz [9] in the 80's. Since then a considerable amount of research has been performed on secure and efficient ECC implementations. The benefits of ECC, when compared with classical cryptosystems such as RSA [23], include: higher speed, lower power consumption and smaller certificates, which are especially useful for wireless applications.

There is a vast literature on differential power analysis (DPA) and differential electromagnetic radiation analysis (DEMA). This paper describes the first DPA and DEMA attack performed on a FPGA implementation of an elliptic curve cryptosystem over $\mathrm{GF}(p)$ [17]. The attacks in previous papers were performed on software implementations or were only simulations of attacks. With the start of differential power analysis in [10], followed by the differential electromagnetic analysis [8,22], several metrics were used to decide for the correct hypothesis. The literature mentions metrics such as the distance of mean test [10], the correlation analysis [18] and the maximum likelihood test [3], all explained in Section 2.2. As we wanted to know which of those yields the best results, we compare them based on the number of measurements needed to obtain the correct key. The number of measurements for the key guess to stabilize is representative for the quality of the metric.

\footnotetext{
${ }^{*}$ Corresponding author.

E-mail address: Siddika.Ors@itu.edu.tr (S.B. Örs).
} 
The paper is structured as follows: In Section 2, the theoretical background of elliptic curves, the power and electromagnetic radiation attacks and the different statistical methods for differential analysis are discussed. Section 3 gives an overview of the previous work in this area. This section is followed by a description of the measurement setup (Section 4). Section 5, describes differential power analysis attack and Section 6 describes differential electromagnetic analysis attack. Section 7 concludes the paper.

\section{Theoretical background}

\subsection{Elliptic curves over $G F(p)$}

An elliptic curve $E$ is expressed in terms of the simplified Weierstrass equation: $y^{2}=x^{3}+a x+b$, where $a$, $b \in \mathrm{GF}(p)$ with $4 a^{3}+27 b^{2} \neq 0(\bmod p)$. The inverse of the point $P=\left(x_{1}, y_{1}\right)$ is $-P=\left(x_{1},-y_{1}\right)$. The sum $P+Q$ of the points $P=\left(x_{1}, y_{1}\right)$ and $Q=\left(x_{2}, y_{2}\right)$ (assume that $P, Q, \neq \mathcal{O}$, and $\left.P \neq \pm Q\right)$ is the point $R=\left(x_{3}, y_{3}\right)$ where: $x_{3}=\lambda^{2}-x_{1}-x_{2}, y_{3}=\left(x_{1}-x_{3}\right) \lambda-y_{1}, \lambda=\frac{y_{2}-y_{1}}{x_{2}-x_{1}}$. For $P=Q$, the "doubling" formulae are: $x_{3}=\lambda^{2}-2 x_{1}, y_{3}=$ $\left(x_{1}-x_{3}\right) \lambda-y_{1}, \lambda=\frac{3 x_{1}^{2}+a}{2 y_{1}}$. The point at infinity $\mathcal{O}$ plays a role analogous to that of the number 0 in ordinary addition. Thus, $P+\mathcal{O}=P$ and $P+(-P)=\mathcal{O}$ for all points $P$. The points on an elliptic curve together with the operation of addition form an Abelian group. Then it is straightforward to introduce the point or scalar multiplication as main operation for elliptic curve cryptosystem (ECC). This operation can be calculated by with the always double-and-add algorithm as shown in Algorithm 1. For details see $[13,9,4]$.

Algorithm 1. Elliptic curve point multiplication (ECPM)

Input: EC point $P=(x, y)$, integer $k, 0<k<M, k=\left(1, k_{l-2}, \ldots, k_{0}\right)_{2}$ and $M$

Output: $Q=[k] P=\left(x^{\prime}, y^{\prime}\right)$

1: $Q \leftarrow P$

2: for $i$ from $l-2$ downto 0

3: $\quad Q_{1} \leftarrow 2 Q$

4: $\quad Q_{2} \leftarrow Q_{1}+P$

5: if $k_{i}=0$ then

6: $\quad Q \leftarrow Q_{1}$

7: else

8: $\quad Q \leftarrow Q_{2}$

\subsection{Power and electromagnetic analysis attacks}

The power consumption of CMOS circuits is data-dependent. However, for the attacker, the relevant question is to know whether this data-dependent behavior is observable.

The first practical implementation of a power analysis attack on the DES was reported in [10] by Kocher et al. Since then, several organizations have developed the skills to conduct these measurements in practice; this now includes knowledge about statistics, the properties of the attacked cryptographic algorithms and the measurement setup.

The current that flows during the transition of the output of a CMOS gate, causes a variation of the electromagnetic field surrounding the chip that can be monitored by inductive probes which are particularly sensitive to the related impulse. The electromotive force across the sensor (Lentz' law) relates to the variation of magnetic flux as follows [25]:

$$
V=-\frac{\mathrm{d} \phi}{\mathrm{d} t} \text { and } \phi=\iint \vec{B} \cdot \mathrm{d} \vec{A}
$$

where $V$ is the probe's output voltage, $\phi$ the magnetic flux sensed by probe, $t$ is the time, $\vec{B}$ is the magnetic field and $\vec{A}$ is the area that it penetrates. 
Maxwell's equation based on Ampère's law relates the magnetic field to their origin:

$$
\vec{\nabla} \times \vec{B}=\mu \vec{J}+\epsilon \mu \frac{\delta \vec{E}}{\delta t}
$$

where $\vec{J}$ is the current density, $\vec{E}$ is the electrical field, $\epsilon$ is the dielectric permittivity and $\mu$ is the magnetic permeability.

Two types of power and electromagnetic analysis attacks are distinguished. In a simple power and electromagnetic analysis (SPA and SEMA) attack, an attacker uses the side-channel information from one measurement directly to determine (parts of) the secret key. In a differential power and electromagnetic analysis (DPA and DEMA) attack, many measurements are used in order to filter out noise. While SPA and SEMA exploits the relationship between the executed operations and the side channel data, DPA and DEMA exploits the relationship between the processed data and the side channel data.

\subsection{Methods for differential analysis}

\subsubsection{Correlation analysis}

In this analysis, the model predicts the amount of side-channel information for a certain moment of the execution. These predictions are correlated to the real side-channel information. The correlation can be measured with the Pearson correlation coefficient[6]. Let $t_{i}$ denote the $i$ th measurement data and $T$ the set of measurements. Let $p_{i}$ denote the prediction of the model for the $i$ th measurement and $P$ the set of such predictions. Then we calculate

$$
C(T, P)=\frac{E(T \cdot P)-E(T) \cdot E(P)}{\sqrt{\operatorname{Var}(T) \cdot \operatorname{Var}(P)}} \quad-1 \leqslant C(T, P) \leqslant 1 .
$$

In Eq. (1), $E(T)$ denotes the expected (average) measurement data of the set of measurements $T$ and $\operatorname{Var}(T)$ denotes the variance of the set of measurements $T$. Note that $T$ and $P$ are said to be uncorrelated, if $C(T, P)$ equals zero; otherwise, they are said to be correlated. If their correlation is high, i.e., if $C(T, P)$ is close to +1 or -1 , it is assumed that the prediction of the model, and thus the key hypothesis, is correct.

\subsubsection{Distance of mean test}

A distance of mean test begins by running the cryptographic algorithm for $N$ random values of input. For each of the $N$ inputs, $I_{i}$, a discrete time side-channel signal, $S_{i}[j]$, is collected and the corresponding output, $O_{i}$, may also be collected. The side-channel signal $S_{i}[j]$ is a sampled version of the side-channel output of the device during the execution of the algorithm that is being attacked. The index $i$ corresponds to the $I_{i}$ that produces the signal and the index $j$ corresponds to the time of the sample. The $S_{i}[j]$ are split into two sets using a partitioning function, $D(\cdot): S_{0}=\left\{S_{i}[j] \mid D(\cdot)=0\right\}, S_{1}=\left\{S_{i}[j] \mid D(\cdot)=1\right\}$.

The next step is to compute the average side-channel signal for each set:

$$
\begin{aligned}
& A_{0}[j]=\frac{1}{\left|S_{0}\right|} \sum_{S_{i}[j] \in S_{0}} S_{i}[j] \\
& A_{1}[j]=\frac{1}{\left|S_{1}\right|} \sum_{S_{i}[j] \in S_{1}} S_{i}[j],
\end{aligned}
$$

where $\left|S_{0}\right|+\left|S_{1}\right|=N$. By subtracting the two averages, a discrete time differential side-channel bias signal, $T[j]$, is obtained: $T[j]=A_{0}[j]-A_{1}[j]$.

Selecting an appropriate $D$ function results in a differential side channel bias signal that can be used to verify guessed part of the secret key.

\subsubsection{Maximum likelihood test}

If $O_{i}, i=1, \ldots, L$ indicates $L$ independent sets of measured signals and if $H_{k}, k=1, \ldots, K$ represents $K$ equally likely hypotheses on some property of these signals, then the maximum likelihood hypothesis test decides in favor of $H_{k}$ if 


$$
k=\arg \max _{1 \leqslant k \leqslant K} \prod_{i=1}^{L} p\left(O_{i} \mid H_{k}\right) .
$$

If we have two hypotheses, we will choose for hypothesis $H_{1}$ if

$$
\prod_{i=1}^{L} p\left(O_{i} \mid H_{1}\right) \geqslant \prod_{i=1}^{L} p\left(O_{i} \mid H_{0}\right)
$$

Assume that $O_{i}$ is a vector of length $n$ and that for all hypotheses the signal has a multivariate Gaussian distribution; under these conditions and taking the natural logarithm of the previous formula, we get that we choose $H_{1}$ when

$$
\sum_{i=1}^{L}\left(\left(O_{i}-\mu_{H_{0}}\right)^{\mathrm{T}} \Sigma_{H_{0}}^{-1}\left(O_{i}-\mu_{H_{0}}\right)-\left(O_{i}-\mu_{H_{1}}\right)^{\mathrm{T}} \Sigma_{H_{1}}^{-1}\left(O_{i}-\mu_{H_{1}}\right)\right) \geqslant L\left(\ln \left|\Sigma_{H_{1}}\right|-\ln \left|\Sigma_{H_{0}}\right|\right)
$$

where $\mu_{H_{i}}, i=0,1$, is the mean value of $H_{i}$. When this theory is used in the traditional distance of mean test and by going over this differentially with the use of a void hypothesis $H_{\mathrm{v}}$, which means using a random division into the 0-bin and the 1-bin, we will decide in favor of $H_{1}$ if $M_{H_{1}} \geqslant M_{H_{0}}$ with

$$
M_{H_{i}}=\frac{\left(\mu_{H_{i}}-E\left[\mu_{H_{\mathrm{v}}}\right]\right)^{2}}{V\left[\mu_{H_{\mathrm{v}}}\right]}-\frac{\left(\mu_{H_{i}}-E\left[\mu_{H_{i}}\right]\right)^{2}}{V\left[\mu_{H_{i}}\right]}-\ln \left(\frac{V\left[\mu_{H_{i}}\right]}{V\left[\mu_{H_{\mathrm{v}}}\right]}\right)
$$

at the correct point in time. The following maximum likelihood estimators are used for the expected values of

the mean and the variance: $E\left[\mu_{H}\right]=\mu_{H}$ and $V\left[\mu_{H}\right]=\frac{\sigma_{H, 0}^{2}}{N_{0}}+\frac{\sigma_{H, 1}^{2}}{N_{1}}$ with $\mu_{H}=\mu_{0}-\mu_{1}$, the difference of the mean of the 0 -bin and the 1-bin, $\sigma_{H_{i}}^{2}$ the variance of the $i$-bin and $N_{i}$ the number of elements in the $i$-bin. Agrawal et al. use this method in [3].

\section{Previous work}

\subsection{Power analysis attacks}

After the description of power-analysis attacks by Kocher et al. [10], the first paper dedicated to the application of these types of attacks to public-key cryptosystems was from Messerges et al. [12]. They studied the application of PA attacks on software implementations of modular exponentiations in smart cards.

Walter et al. observe in [27] that also modular subtractions can be used to determine the secret key. In [26] Walter shows, how to attack an RSA secret key without multiple measurements and additionally he shows that also certain implementations of sliding window techniques might be vulnerable to PA attacks.

Coron's work [7] was the first article on PA attacks dedicated solely to ECC. A conclusion from this paper is that implementations of a static elliptic curve Diffie-Helman (ECDH) and an elliptic curve integrated encryption scheme (ECIES) protocol should be made resistant to power analysis attacks.

Nguyen and Shparlinski pointed out that also the scalar point-multiplication in the elliptic curve digital signature algorithm (ECDSA) must resist PA attacks in [14]. The paper gives a rigorous treatment of this topic. Romer and Seifert treated the same topic with more heuristic arguments in a similar paper [24].

Oswald has developed another type of attack in [20], to attack countermeasures which are based on randomizing (variants of) the binary algorithm. This attack can defeat countermeasures that randomize the sequence of instructions in the binary algorithm in a certain way [21] under the assumption that the power trace from an EC point-addition operation can be distinguished from the power trace of an EC point-doubling operation. Okeya and K. Sakurai mounted another type of attack to defeat certain variants of these randomization approaches in [16]. It assumes that an attacker can monitor several executions of an EC scalar point-multiplication with the same scalar and it additionally assumes that EC point-addition and EC point-doubling can be distinguished.

\subsection{Electromagnetic analysis attacks}

It is well-known that the US government has been aware of electromagnetic leakage since the 1950's. The resulting standards are called TEMPEST; partially declassified documents can be found in [15]. The first pub- 
lished papers are work of Quisquater and Samyde [22] and the Gemplus team [8]. Quisquater and Samyde showed that it is possible to measure the electromagnetic radiation from a smart card. Their measurement setup consisted of a sensor which was a simple flat coil, a spectrum analyzer or an oscilloscope and a Faraday cage. Quisquater also introduced the terms simple EMA (SEMA) and Differential EMA (DEMA). The work of Gemplus deals with experiments on three algorithms: DES, RSA and COMP128. They observed the feasibility of EMA attacks and compared them with power analysis attacks in favor of the first.

According to Agrawal et al. there are two types of emanations: intentional and unintentional [2,1]. The first type results from direct current flows. The second type is caused by various couplings, modulations (AM and FM), etc. The two papers mentioned above deal exclusively with intentional emanations. To the contrary, the real advantage over other side-channel attacks lies in exploring unintentional emanations [2,1]. More precisely, EM leakage consists of multiple channels. Therefore, compromising information can be available even for DPA resistant devices which can be detached from the measurement equipment.

Namely, besides carefully exploring all available EM emanations an attacker can also focus on a combination of two or more side-channels. Agrawal et al. defined these so-called multi-channel attacks in which the side-channels are not necessarily of a different kind [3]. For example, they discussed combined power and EM analysis but also multi-channel DPA attacks. The former uses a CMOS leakage model and the maximum-likelihood principle for performing and analyzing.

Mangard also showed that near-field EM attacks can be conducted even with a simple hand-made coil in [11]. Besides that he showed that measuring the far-field emissions of a smart card connected to a power supply unit also suffices to determine the secret key used in the smart card.

Carlier et al. showed that EM side channels from an FPGA implementation of AES can be effectively used by an attacker to retrieve some secret information in [5]. They worked close to the FPGA and by this way were able to get rid of the effects of other computations made at the same time.

Up to now, most papers on EMA applied similar techniques as power analysis while apparently much more information is available to be explored. It is likely that future work will also deal with combinations of EMA with other side channel attacks.

\section{Measurement setup}

The measurement setup consists of the FPGA board with a Xilinx Virtex 800 FPGA presented in [19], an Tektronix TDS714L oscilloscope, a Tektronix CT1 current probe, a handmade loop antenna, a function generator and a power supply. The total power consumption and the electromagnetic radiation of the FPGA were measured simultaneously while it executes an elliptic curve point multiplication with the key and a point on the curve.

\section{DPA Attack on an FPGA implementation of an elliptic curve cryptosystem over GF(p)}

In this section, we conduct a DPA attack on a FPGA implementation of an elliptic curve processor over $\mathrm{GF}(p)$ [17]. The current consumption trace of one EC point multiplication is shown in Fig. 2a.

The target for our DPA attack is the second most significant bit (MSB) of the key, $k_{l-2}$, in Algorithm 1. There are two temporary point registers in the design, $Q_{1}$ and $Q_{2}$. These temporary points and the output point $Q$ are updated in the following order.

Step 1: $Q \leftarrow P$

Step 3: $Q_{1} \leftarrow 2 Q=2 P$

Step 4: $Q_{2} \leftarrow Q_{1}+P=3 P$

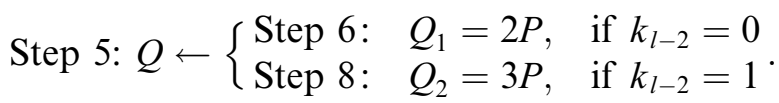

Step 3: $Q_{1} \leftarrow\left\{\begin{array}{ll}2 Q=4 P, & \text { if } k_{l-2}=0 \\ 2 Q=6 P, & \text { if } k_{l-2}=1\end{array}\right.$. 


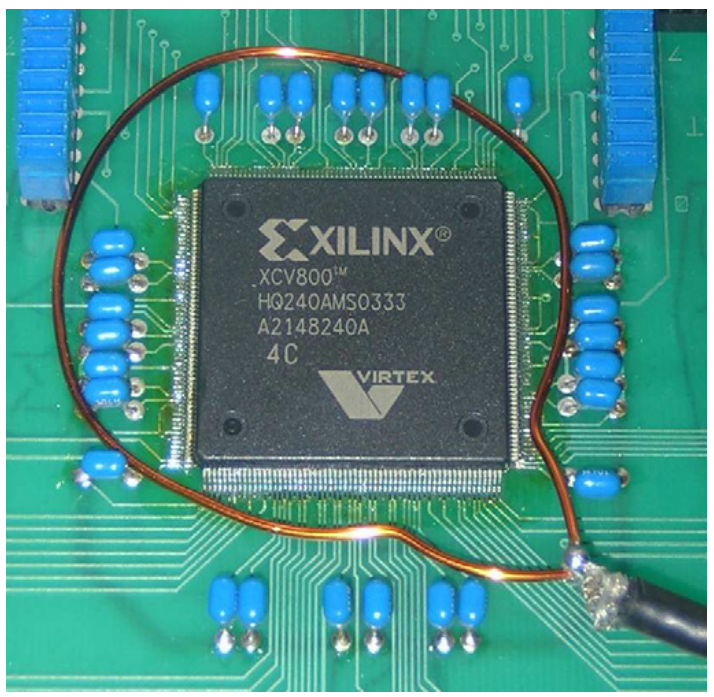

Fig. 1. Measurement setup.
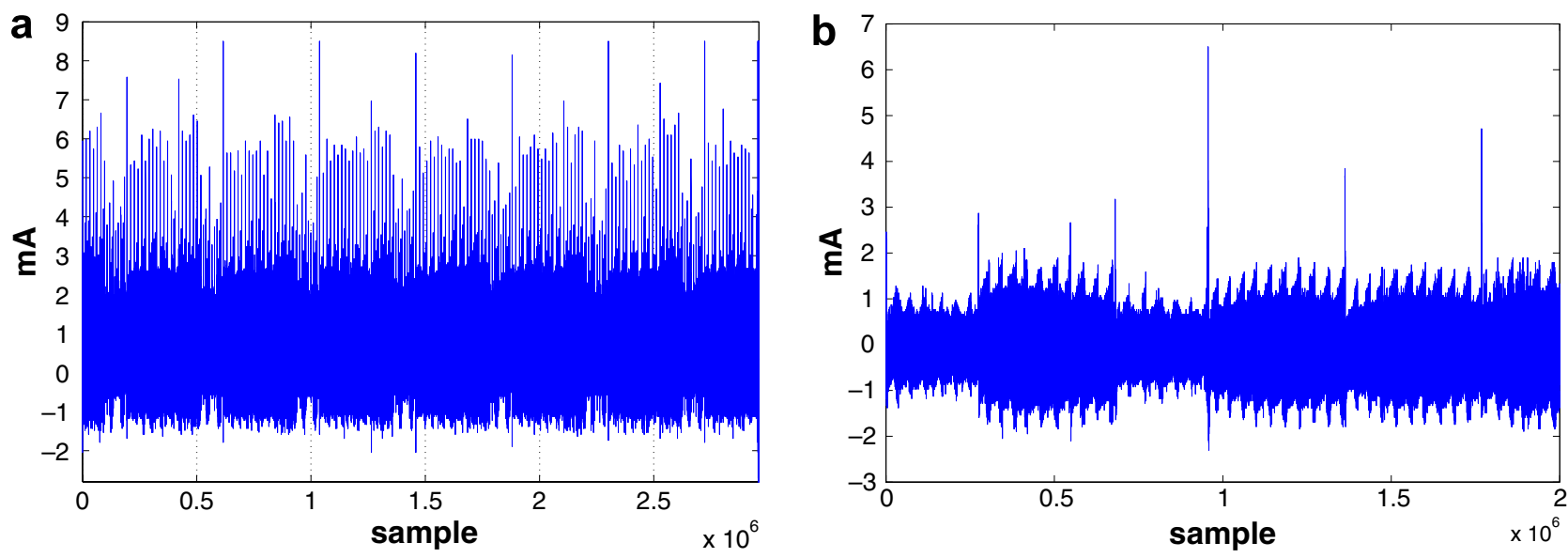

Fig. 2. Current consumption trace of a 160-bit ECPM over GF(p) with Algorithm 1: (a) complete and (b) around the attack point.

The first step of the DPA attack is to find the point to measure. The current consumption trace of an EC point multiplication is shown in Fig. 2. The highest seven spikes on Fig. 2 show the end of seven EC point doubling operations. Because these spikes are clearly higher than other spikes on the current consumption trace, our attack point is one of these seven spikes. The first one corresponds to the end of the first EC doubling operation. As shown above this spike shows the end of the second operation which is $Q_{1} \leftarrow 2 P$ and this step is executed independently from the key bits. The third, fourth and later spikes need the knowledge of the $k_{l-2}, k_{l-3}$ etc. Hence our choice for the measurement point is the second update of $Q_{1}$ after the second EC point doubling (Step 3). We use the transitions between the previous value of $Q_{1}, 2 P$, and the new value at our target point, $4 P$ or $6 P$ according to the value of $k_{l-2}$ as the power consumption predictions.

\subsection{Correlation analysis}

In the first step of our attack, we have produced a power consumption file. For this purpose, we have chosen $N$ random points on the EC and one fixed, but random key, $k$. The FPGA executes $N$ point multiplications such that $Q_{i}=[k] P_{i}$ for $i=1,2, \ldots, N$. We have measured the power consumption of the FPGA during 2400 
clock cycles around the second update of $Q_{1}$. The clock frequency applied to the chip was around $300 \mathrm{kHz}$ and the sampling frequency of the oscilloscope was $250 \mathrm{MHz}$. With these measurements, we have produced a $N \times$ 2,000,000 matrix, $M_{1}$. The current consumption trace of one of these measurements, is shown in Fig. $2 \mathrm{~b}$.

We have applied a pre-processing technique to reduce the amount of measurement data in every clock cycle. We have found the maximum value of the measurement data in each clock cycle as $M_{2}(i, j)=$ $\max \left(M_{1}\left(i, D_{i} \cdot(j-1)+1: D_{i} \cdot j\right)\right)$, where $i=1, \ldots, N, j=1, \ldots, 2400 . M_{2}(i, j)$ is the element of the matrix $M_{2}$ at the $i$ th row and the $j$ th column. $D_{i}$ is the number of samples per clock cycle during $i$ th measurement, $M_{1}\left(i, D_{i} \cdot(j-1)+1: D_{i} \cdot j\right)$ is the row vector $\left[M_{1}\left(i, D_{i} \cdot(j-1)+1\right) M_{1}\left(i, D_{i} \cdot(j-1)+2\right) \ldots M_{1}\left(i, D_{i} \cdot j\right)\right]$.

Because the clock frequency of the function generator in our experiments was varying slightly during the measurements we have to find $D_{i}$. In order to compute $D_{i}$ we have to know the exact clock frequency. We have calculated the discrete Fourier transform (DFT) of the $i$ th measurement, corresponding the $i$ th row of $M_{1}$. As the clock frequency can vary between $250 \mathrm{kHz}$ and $375 \mathrm{kHz}$, we have searched between these frequencies for the maximum value in the DFT trace. The result for the first measurement is shown in Fig. 3. According to this figure the clock frequency during the first measurement was $302.8 \mathrm{kHz}$. Hence, $D_{1}=250 \times 10^{6} /$ $302.8 \times 10^{3}=825.63$. Fig. 4 shows the first measurement after taking the maximum value in every clock cycle.

We have implemented the EC point multiplication with Algorithm 1 in the C programming language. The C program computes $N$ EC point multiplications with $N$ EC points and the key. The EC points and the key are the same as the ones given to the FPGA during the measurements. During the execution of the EC point multiplications, the $\mathrm{C}$ program computes the number of bits that change from 0 to 1 in some registers at the

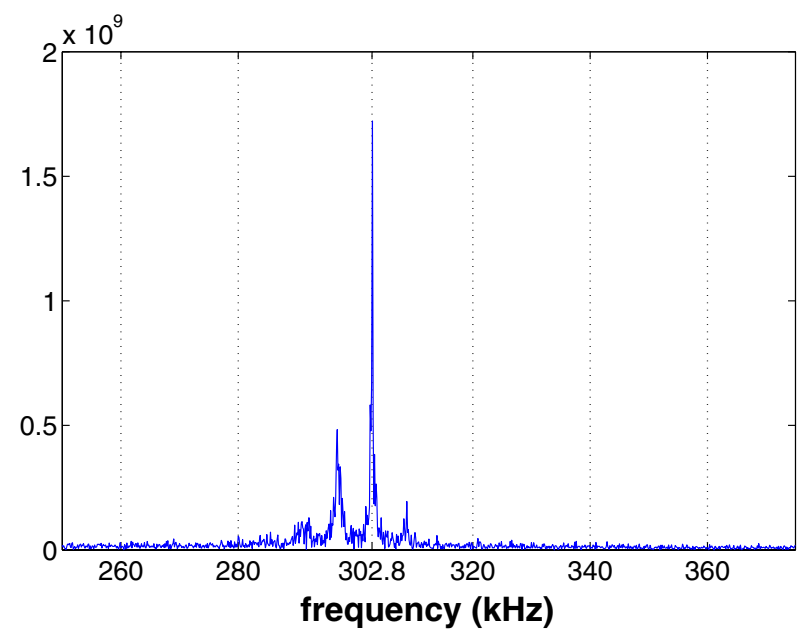

Fig. 3. DFT of the current consumption trace of a measurement between $250 \mathrm{kHz}$ and $375 \mathrm{kHz}$.

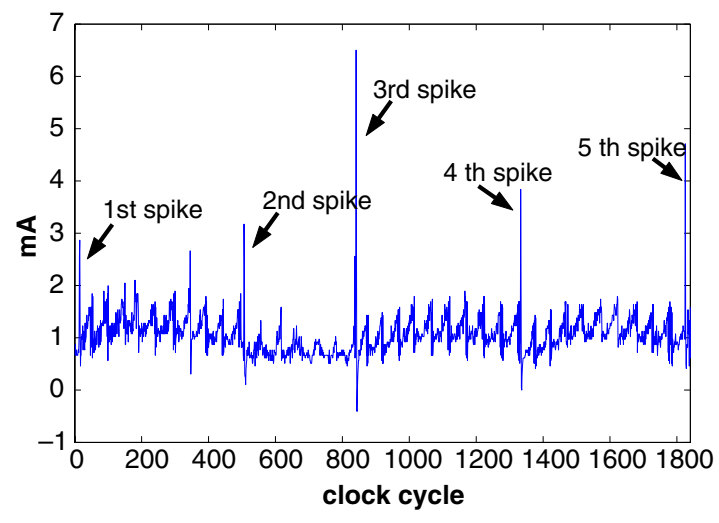

Fig. 4. Current consumption trace of a measurement after taking the maximum value in every clock cycle. 
steps corresponding to the five spikes shown in Fig. 4. The number of transitions is used as the power consumption prediction.

One of the steps of the attack is to find the right steps in the $\mathrm{C}$ program to predict the power consumption which corresponds to the measurement points. As we want to measure the power consumption of the FPGA around the last clock cycle of the second EC doubling in Step 3 of Algorithm 1, the third spike in Fig. 4 corresponds to this event. Hence we can predict it by counting the number of transitions between the bits of the coordinates of $Q_{1}$ and $Q_{2}$; for $k_{l-2}=0$ we count the number of transitions between $2 P$ and $4 P$ and for $k_{l-2}=1$ between $2 P$ and $6 P$.

We have produced two power consumption prediction matrixes, $M_{3}$ and $M_{4}$, for the $k_{l-2}=0$ and $k_{l-2}=1$ guesses, respectively. $M_{3}$ and $M_{4}$ have one column for the third spike and $N$ rows for the $N$ EC points.

Now we can learn the right value of $k_{l-2}$ by finding the correlations between $M_{3}$ and $M_{4}$ and the column of $M_{2}$ which corresponds to the third spike in Fig. 4. If the correlation between $M_{3}$ and $M_{2}$ is higher than the correlation between $M_{4}$ and $M_{2}$, then we decide that $k_{l-2}=0$, otherwise we decide that $k_{l-2}=1$. We are also interested in finding the minimum number of measurements that are necessary to find the right key-bit. Fig. 5 shows the change in correlation between $M_{3}$ and $M_{4}$ and the column of $M_{2}$ which corresponds to the third spike in Fig. 4 according to the number of measurements used.

It is visible from Fig. 5 that the correlation for the prediction for the $k_{l-2}=1$ guess is higher than the correlation for the prediction for the $k_{l-2}=0$ guess. By using the first 4000 measurements the decision of $k_{l-2}=1$ can be made.

\subsection{Distance of mean test}

We produce a new measurement matrix, $M_{5}$, from $M_{2}$ by taking 20 columns around each column of $M_{2}$ that corresponds to a spike in Fig. 4 . Thus, $M_{5}$ has 100 columns and $N$ rows. We use the prediction matrices $M_{3}$ (for $k_{l-2}=0$ guess) and $M_{4}$ (for $k_{l-2}=1$ guess) explained in Section 5.1 in order to split the measurements into sets. There are two sets for the $k_{l-2}=0$ guess and the $k_{l-2}=1$ guess. We calculate the mean value of $M_{3}$ and $M_{4}, E\left(M_{3}\right)$ and $E\left(M_{4}\right)$, respectively. If the prediction for the $j$ th $(j=1,2, \ldots, 10,000)$ measurement for $k_{l-2}=0$ guess is smaller than $E\left(M_{3}\right)\left(M_{3}(j)<E\left(M_{3}\right)\right)$ then $j$ th measurement is put in set $S_{1,1}$, otherwise in set $S_{1,2}$. If the prediction for the $j$ th $(j=1,2, \ldots, 10,000)$ measurement for $k_{l-2}=1$ guess is smaller than $E\left(M_{4}\right)\left(M_{4}(j)<E\left(M_{4}\right)\right)$ then $j$ th measurement is put in set $S_{2,1}$, otherwise in set $S_{2,2}$. Then we calculate the mean values for each of the sets and find the bias signal for the $j$ th guess as $T_{j}=E\left(S_{j, 2}\right)-E\left(S_{j, 1}\right)$. The current consumption bias signals for $k_{l-2}=0$ and $k_{l-2}=1$ guesses are shown in Fig. 6 . The figure shows a high peak on the expected spot on the line for the $k_{l-2}=1$ guess. Hence the decision for the right key-bit is again equal to 1 .

In order to compare the correlation analysis and distance of mean test we should also find the number of measurements needed to find the right key bit for the distance of mean test. Fig. 7 shows the change in the

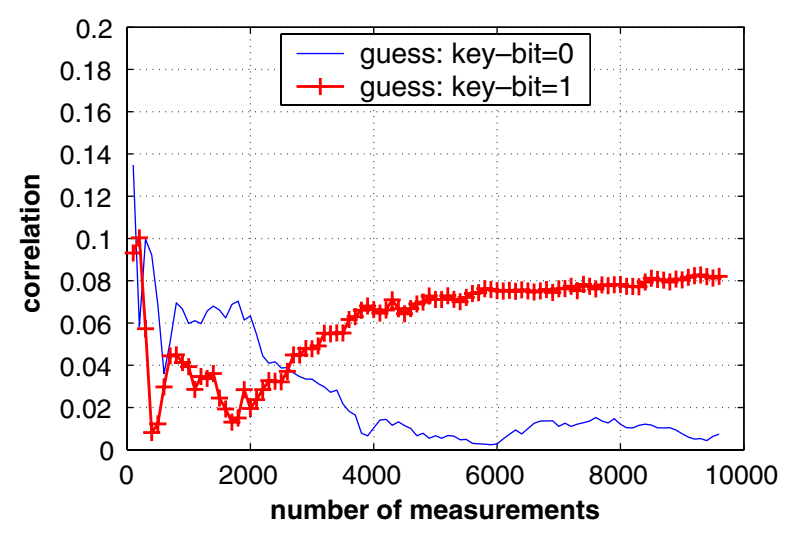

Fig. 5. Correlation between the current consumption measurements and the predictions of the third spike in Fig. 4 as a function of the number of measurements. 


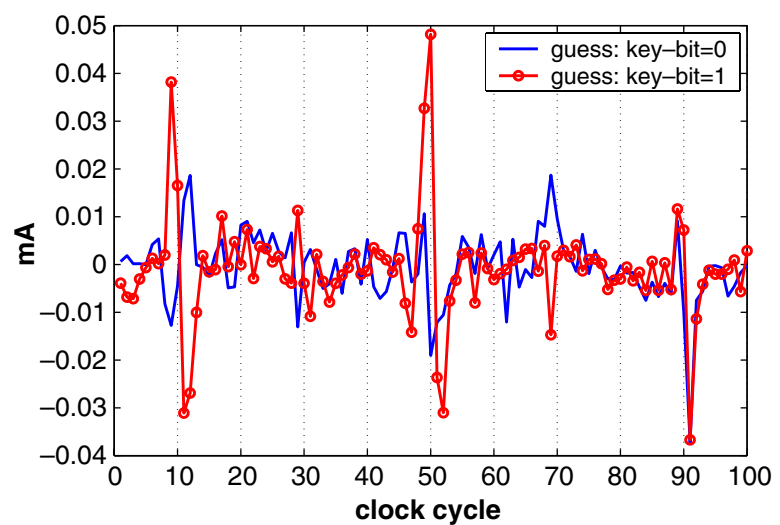

Fig. 6. Current consumption bias signals for the third spike in Fig. 4 and the $k_{l-2}=0$ and $k_{l-2}=1$ guesses.

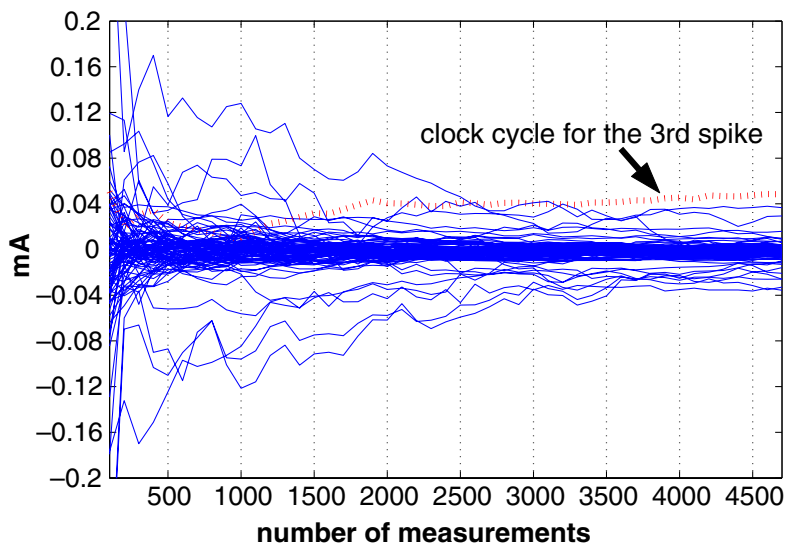

Fig. 7. Change in the amplitude of the current consumption bias signal for the third spike in Fig. 4 , the $k_{l-2}=1$ guess and for all clock cycles.

amplitude of all the clock cycles on the current consumption bias signals for the $k_{l-2}=1$ guess. The number of measurements on these traces are the number of measurements in the sets $S_{2,1}, S_{2,2}$ described above. The number of measurements in these sets are nearly the same. Hence we should multiply the number of measurements seen in Fig. 7 by two in order to find the needed number of measurements. As it is shown in Fig. 7, 9000 measurements are needed to distinguish the right clock cycle from the wrong ones. When we compare the results shown in Figs. 5 and 7, we conclude that we need approximately two times more measurements for the distance of mean test than for correlation analysis.

\subsection{Maximum likelihood test}

We reuse the measurement matrix, $M_{5}$, from Section 5.2. We use the prediction matrices $M_{3}$ (for $k_{l-2}=0$ guess) and $M_{4}$ (for $k_{l-2}=1$ guess) explained in Section 5.1 in order to split the measurements into sets. There are again two sets for the $k_{l-2}=0$ guess and the $k_{l-2}=1$ guess produced in the same way as in the previous section. Then we calculate (2) for each guess of key-bit and compare it against each other. The current consumption bias signals for $k_{l-2}=0$ and $k_{l-2}=1$ guesses are shown in Fig. 8. The figure shows a high peak on the expected spot on the line for the $k_{l-2}=1$ guess. Hence the decision for the right key-bit is again 1 .

In order to compare with the other metrics we should again find the number of measurements needed to find the right key-bit for the maximum likelihood test. Fig. 9 shows the change in the amplitude of all the clock cycles on the current consumption bias signals for the $k_{l-2}=1$ guess. As it is shown in Fig. 9, 5000 measurements are needed to distinguish the right clock cycle from the wrong ones. When we compare the results 


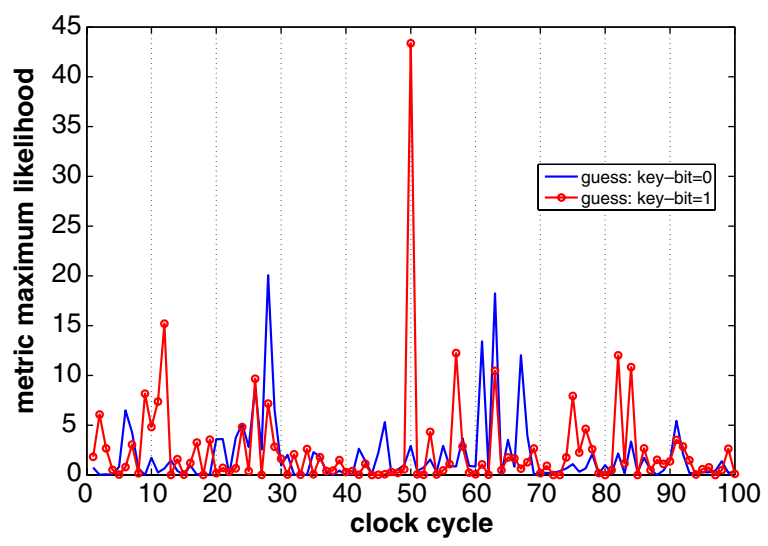

Fig. 8. The metric for the maximum likelihood test for the third spike in Fig. 4 and the $k_{l-2}=0$ and $k_{l-2}=1$ guesses.

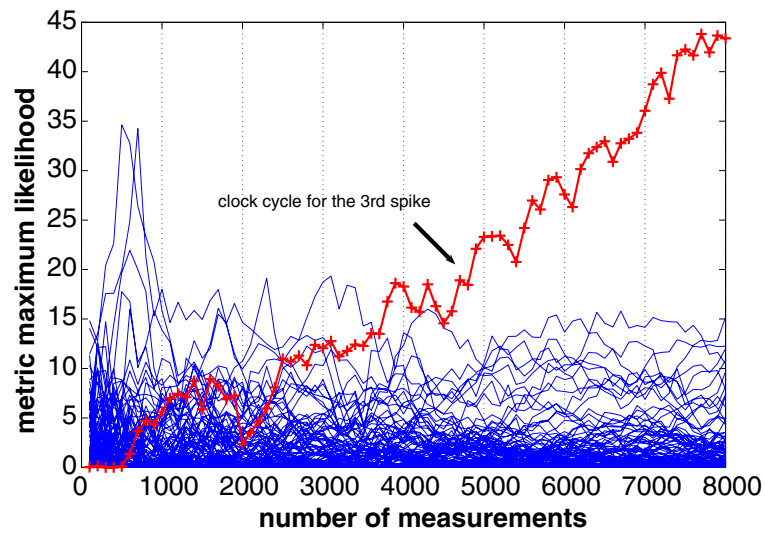

Fig. 9. Change in the amplitude of the metric of maximum likelihood for the third spike in Fig. 4, the $k_{l-2}=1$ guess and for all clock cycles.

shown in Figs. 5, 7 and 9, we conclude that the maximum likelihood test on the power measurements needs more or less the same number of measurements as the correlation analysis but twice fewer as the distance of mean test.
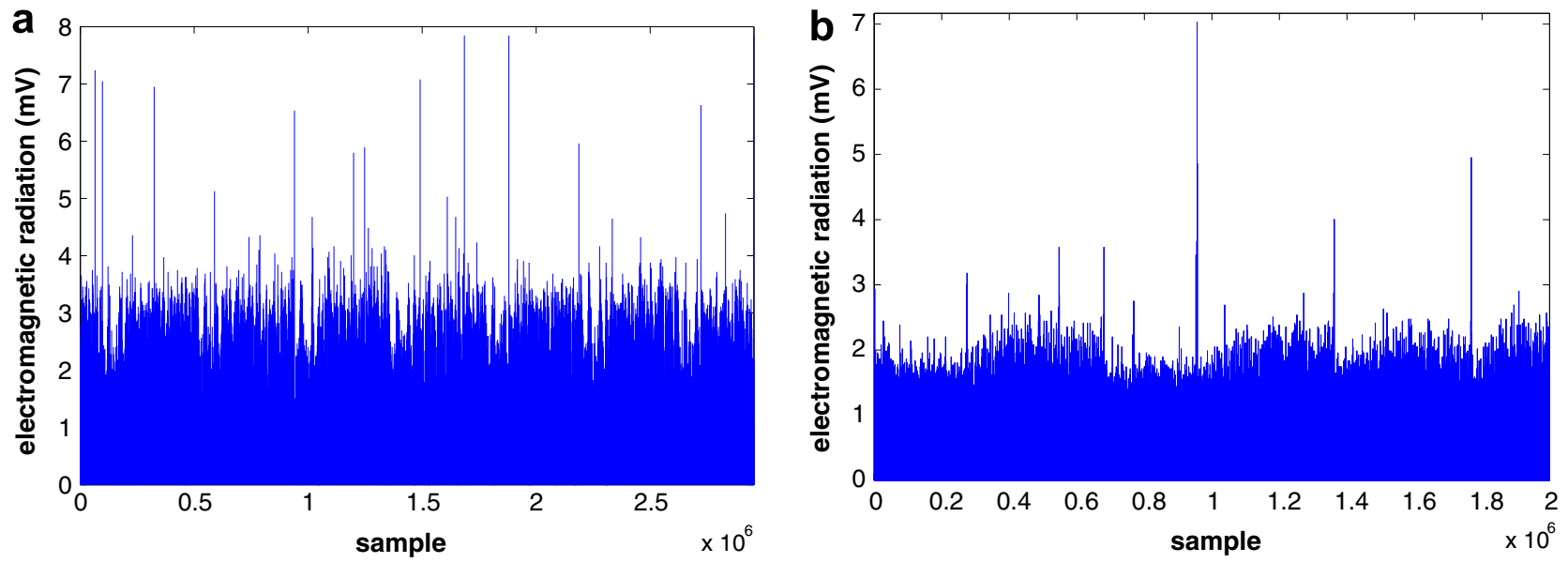

Fig. 10. Electromagnetic radiation trace of a 160-bit ECPM with Algorithm 1: (a) complete and (b) around the attack point. 


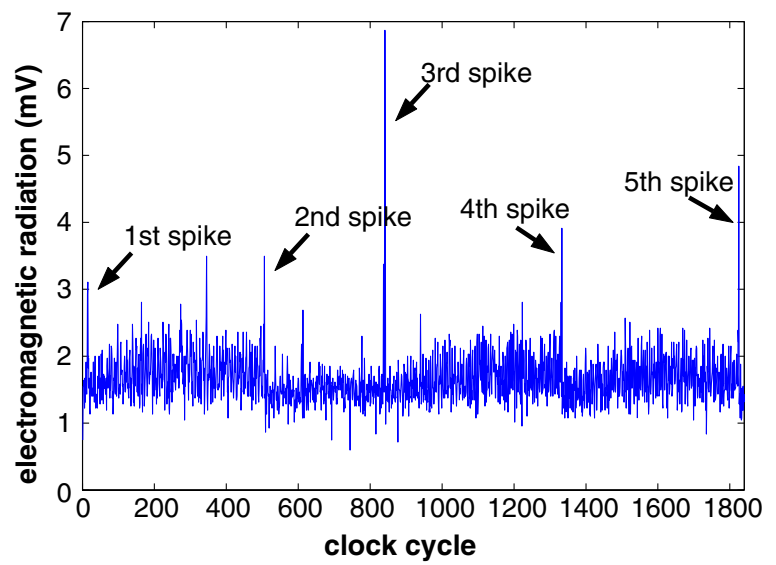

Fig. 11. Electromagnetic radiation trace of a measurement after taking the maximum value in every clock cycle.

\section{DEMA attack on an FPGA implementation of an elliptic curve cryptosystem over GF(p)}

In this section, we conduct a DEMA attack on a FPGA implementation of our elliptic curve processor over $\mathrm{GF}(p)$. One EM measurement of this architecture is shown in Fig. 10a. The target for our DEMA attack is the second MSB of the key, $k_{l-2}$, in Algorithm 1.

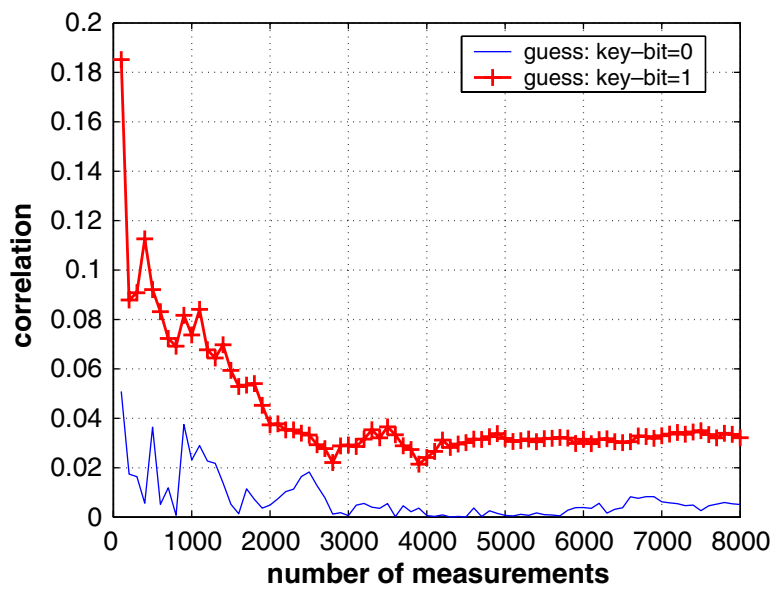

Fig. 12. Correlations between the electromagnetic radiation measurements and the predictions of the third spike in Fig. 11 as a function of the number of measurements.

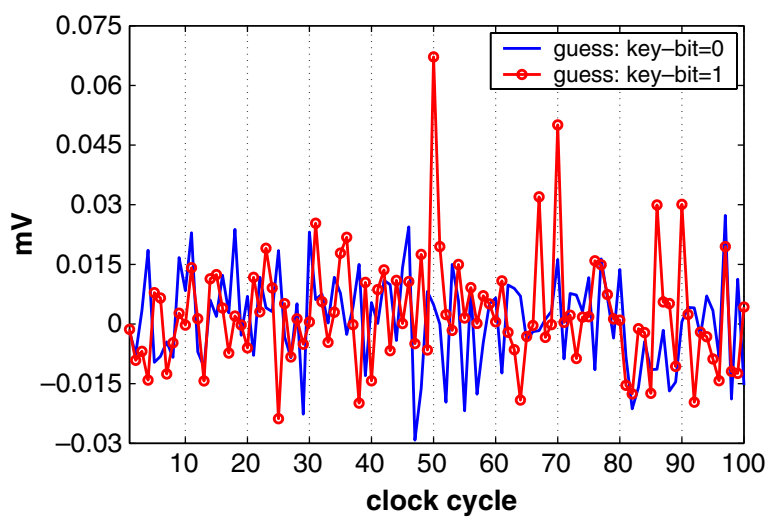

Fig. 13. Electromagnetic radiation bias signals for the third spike in Fig. 11 and the $k_{l-2}=0$ and $k_{l-2}=1$ guesses. 


\subsection{Correlation analysis}

In the first step of our attack, we have produced an electromagnetic radiation file. For this purpose, we have measured the electromagnetic radiation at the same time as the power consumption measurements given in Section 5.1 and produced $M_{1}$ in the same way. The electromagnetic radiation trace of one of these measurements is shown in Fig. 10b.

We have applied the same pre-processing technique as in Section 5.1 to the data from the oscilloscope. Fig. 11 shows the 1st measurement after taking the maximum value in every clock cycle.

We have predicted the electromagnetic radiation of the FPGA during the events which correspond to the third spike shown in Fig. 11 for $k_{l-2}=0$ and $k_{l-2}=1$ guesses as Fig. 12 indicates that the correlation for the prediction for the $k_{l-2}=1$ guess is higher than the correlation for the prediction for the $k_{l-2}=0$ guess. By just using the first 2000 measurements the decision of $k_{l-2}=1$ can be made.

\subsection{Distance of mean test}

The EM radiation measurements are split into the sets with the partitioning function as defined in Section 5.2. The electromagnetic bias signals for guesses $k_{l-2}=0$ and $k_{l-2}=1$ are shown in Fig. 13. The figure shows a high peak on the expected spot on the line for the $k_{l-2}=1$ guess in Fig. 13. Hence the decision for the right key bit is again 1.

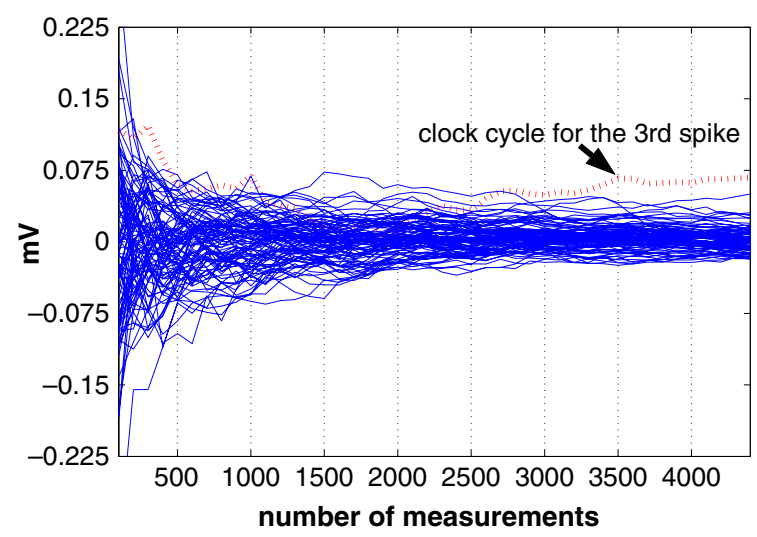

Fig. 14. Change in the amplitude of the electromagnetic radiation bias signal for the third spike in Fig. 11 , the $k_{l-2}=1$ guess and for all clock cycles.

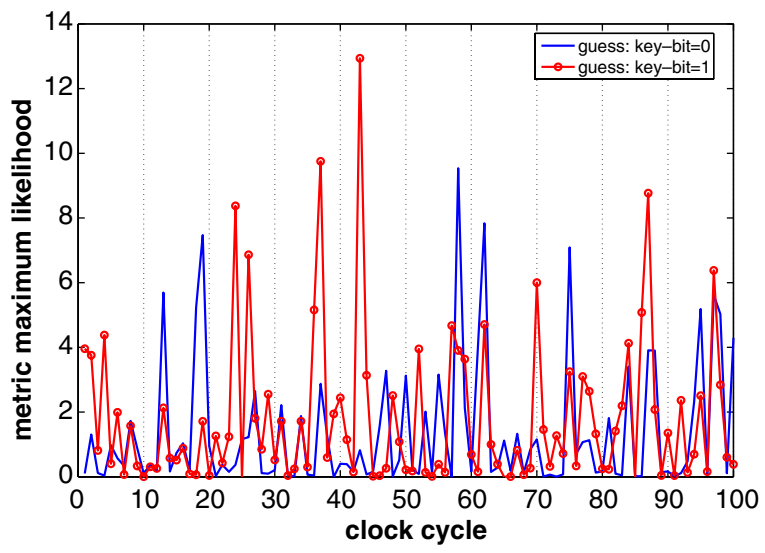

Fig. 15. The metric for the maximum likelihood test for the third spike in Fig. 11 and the $k_{l-2}=0$ and $k_{l-2}=1$ guesses. 


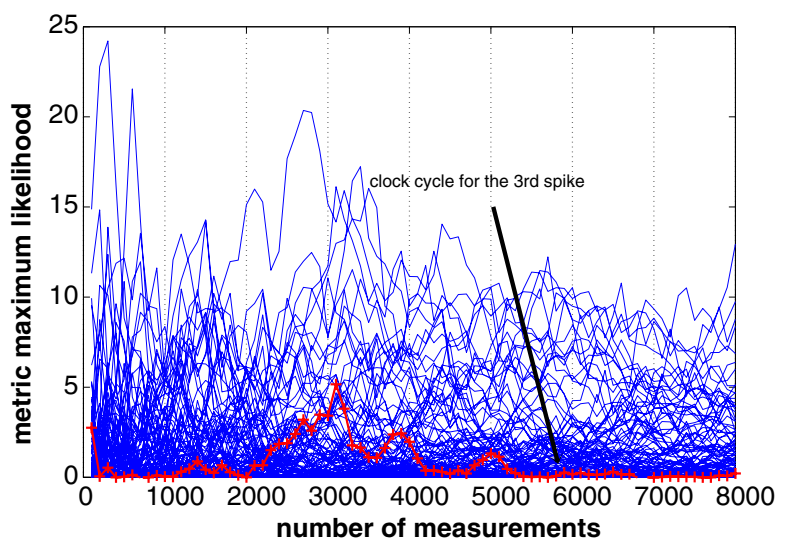

Fig. 16. Change in the amplitude of the metric for the maximum likelihood test for the third spike in Fig. 11 , the $k_{l-2}=1$ guess and for all clock cycles.

Again we find the number of measurements needed to find the right key bit for distance of mean test. Fig. 14 shows the change in the amplitude of all the data points on the EM radiation bias signals for the $k_{l-2}=1$ guess. The right clock cycle is visible by using 7000 measurements in Fig. 14. When we compare the results shown in Figs. 12 and 14, we should use five times more measurements for the distance of mean test than for correlation analysis.

\subsection{Maximum likelihood test}

The same processing technique as for the power measurements in Section 5.3 is applied to the set of electromagnetic radiation measurements. The result is shown in Fig. 15. The figure shows no high peak on the expected spot on the line for the $k_{l-2}=1$ guess in Fig. 15. Therefor a decision on the right key-bit can not be made. The right clock cycle is not visible by using the maximum likelihood test in Fig. 16. The reason for this can be that using the same prediction model for power and electromagnetic radiation is not always a good choice or that the amount of measurements we took is insufficient.

\section{Conclusions}

We have implemented differential power and electromagnetic analysis attacks on an FPGA implementation of elliptic curve cryptosystems over $\mathrm{GF}(p)$. We use three well-known techniques for DPA and DEMA: correlation analysis, a distance of mean test and a maximum likelihood test. The following table summarizes the number of measurements needed for each analysis technique.

From Table 1, we conclude that correlation analysis reveals the right key bit by using two times fewer measurements than the distance of mean test for DPA and three times fewer for DEMA. The number of required electromagnetic radiation measurements is less than the power consumption measurements in order to find the right key bit for the correlation analysis and distance of mean test, but the maximum likelihood test does not give us the right key-bit with a DEMA attack.

This result can be explained as follows. We use a very simple handmade antenna shown in Fig. 1. Because the diameter of the antenna is large and it is not isolated from any effect by any protection, the antenna collects all the signals in the air. We use the same prediction data for both power consumption and electromagnetic radiation, which is the number of transitions in the target registers. This is a correct model for power

Table 1

Number of measurements needed to find the correct key for the different differential analysis techniques

\begin{tabular}{llll}
\hline & Correlation analysis & Distance of mean & Maximum likelihood \\
\hline Power & 4000 & 9000 & 5000 \\
Electromagnetic radiation & 2000 & 7000 & unknown \\
\hline
\end{tabular}


consumption as it is related to the current flow to the load capacitances, but the electromagnetic radiation is also effected by the direction of the current internally and the surrounding electrical field. So the predictions for the electromagnetic radiation should be improved by taking into account some more parameters, among them the position of the antenna on the FPGA and the relative direction of the current flows.

\section{Acknowledgements}

This work is supported by Institute for the Promotion of Innovation through Science and Technology in Flanders and FWO G.0475.05 Projects.

\section{References}

[1] Agrawal D, Archambeault B, Chari S, Rao JR, Rohatgi P. Advances in side-channel cryptanalysis. RSA Lab Cryptobytes 2003;6(1):20-32.

[2] Agrawal D, Archambeault B, Rao JR, Rohatgi P. The EM side-channel(s): attacks and assessment methodologies. In: Kaliski Jr BS, Koç ÇK, Paar C, editors. Proceedings of the fourth international workshop on cryptographic hardware and embedded systems (CHES). Lecture notes in computer science, vol. 2523. Redwood Shores, CA, USA: Springer-Verlag; 2002.

[3] Agrawal D, Rao JR, Rohatgi P. Multi-channel attacks. In: Walter C, Koç ÇK, Paar C, editors. Proceedings of the fifth international workshop on cryptographic hardware and embedded systems (CHES). Lecture notes in computer science, vol. 2779. Cologne, Germany: Springer-Verlag; 2003.

[4] Blake I, Seroussi G, Smart NP. Elliptic curves in cryptography. London mathematical society lecture note series. Cambridge: Cambridge University Press; 1999.

[5] Carlier V, Chabanne H, Dottax E, Pelletier H. Electromagnetic side channels of an FPGA implementation of AES, Cryptology ePrint Archive-2004/14; 2004. <http://eprint.iacr.org/>.

[6] Clarke GM, Cooke D. A basic course in statistics. fourth ed. London: Arnold; 1998.

[7] Coron J-S. Resistance against differential power analysis for elliptic curve cryptosystems. In: Koç ÇK, Paar C, editors. Proceedings of the first international workshop on cryptographic hardware and embedded systems (CHES). Lecture notes in computer science, vol. 1717. Worcester, MA, USA: Springer-Verlag; 1999.

[8] Gandolfi K, Mourtel C, Olivier F. Electromagnetic analysis: concrete results. In: Koç ÇK, Naccache D, Paar C, editors. Proceedings of third international workshop on cryptographic hardware and embedded systems (CHES). Lecture notes in computer science, vol. 2162. Paris, France: Springer-Verlag; 2001.

[9] Koblitz N. Elliptic curve cryptosystem. Math Comp 1987;48:203-9.

[10] Kocher P, Jaffe J, Jim B. Differential power analysis. In: Wiener M, editor. Advances in cryptology: proceedings of CRYPTO’99. Lecture notes in computer science, vol. 1666. Santa Barbara, CA, USA: Springer-Verlag; 1999.

[11] Mangard S. Exploiting radiated emissions - EM attacks on cryptographic ICs. In: Proceedings of Austrochip, Linz, Austria; 2003.

[12] Messerges TS, Dabbish EA, Sloan RH. Power analysis attacks of modular exponentiation in smartcards. In: Koç ÇK, Paar C, editors. Proceedings of the first international workshop on cryptographic hardware and embedded systems (CHES). Lecture notes in computer science, vol. 1717. Worcester, MA, USA: Springer-Verlag; 1999.

[13] Miller V. Uses of elliptic curves in cryptography. In: Williams HC, editor. Advances in cryptology: proceedings of CRYPTO'85. Lecture notes in computer science, vol. 218. Santa Barbara, CA, USA: Springer-Verlag; 1985.

[14] Nguyen PQ, Shparlinski IE. The insecurity of the elliptic curve digital signature algorithm with partially known nonces. Desig Codes Cryptograph 2003;30(2):201-17.

[15] NSA, NSA TEMPEST Documents; 2003. <http://www.cryptome.org/nsa-tempest.htm>.

[16] Okeya K, Sakurai K. On insecurity of the side channel attack countermeasure using addition-subtraction chains under distinguishability between addition and doubling. In: Batten LM, Seberry J, editors. Proceedings of the seventh Australian conference information security and privacy (ACISP). Lecture notes in computer science, vol. 2384. Melbourne, Australia: SpringerVerlag; 2002.

[17] Ors SB, Batina L, Preneel B, Vandewalle J. Hardware implementation of an elliptic curve processor over GF( $p$ ). In: IEEE 14th international conference on application-specific systems. The Hague, The Netherlands: Architectures and Processors (ASAP); 2003.

[18] Ors SB, Gürkaynak FK, Oswald E, Preneel B. Power-analysis attack on an ASIC AES implementation. In: Proceedings of the international conference on information technology (ITCC), Las Vegas, NV, USA; 2004.

[19] Ors SB, Oswald E, Preneel B. Power-analysis attacks on an FPGA - first experimental results. In: Walter C, Koç ÇK, Paar C, editors. Proceedings of the fifth international workshop on cryptographic hardware and embedded systems (CHES). Lecture notes in computer science, vol. 2779. Cologne, Germany: Springer-Verlag; 2003.

[20] Oswald E. Enhancing simple power-analysis attacks on elliptic curve cryptosystems. In: Kaliski Jr BS, Koç ÇK, Paar C, editors. Proceedings of the fourth international workshop on cryptographic hardware and embedded systems (CHES). Lecture notes in computer science, vol. 2523. Redwood Shores, CA, USA: Springer-Verlag; 2002.

[21] Oswald E, Aigner M. Randomized addition-subtraction chains as a countermeasure against power attacks. In: Koç ÇK, Naccache D, Paar C, editors. Proceedings of the third international workshop on cryptograpic hardware and embedded systems (CHES). Lecture notes in computer science, vol. 2162. Paris, France: Springer-Verlag; 2001. 
[22] Quisquater J-J, Samyde D. Electromagnetic analysis (EMA): measures and counter-measures for smard cards. In: Attali I, Jensen T, editors. Proceedings of the international conference on research in smart cards: smart card programming and security (E-smart). Lecture notes in computer science, vol. 2140. Cannes, France: Springer-Verlag; 2001.

[23] Rivest RL, Shamir A, Adleman L. A method for obtaining digital signatures and public-key cryptosystems. Commun ACM 1978;21(2):120-6.

[24] Romer T, Seifert J-P. Information leakage attacks against smart card implementations of the elliptic curve digital signaturealgorithm. In: Attali I, Jensen TP, editors. Proceedings of the international conference on research in smart cards, smart card programming and security (E-smart). Lecture notes in computer science, vol. 2140. Cannes, France: Springer-Verlag; 2001.

[25] Serway RA. Physics for scientists and engineers. Saunders golden sunburst series. Saunders college publishing; 1996.

[26] Walter CD. Sliding windows succumbs to big mac attack. In: Koç ÇK, Naccache D, Paar C, editors. Proceedings of third international workshop on cryptographic hardware and embedded systems (CHES). Lecture notes in computer science, vol. 2162. Paris, France: Springer-Verlag; 2001.

[27] Walter CD, Thompson S. Distinguishing exponent digits by observing modular subtractions. In: Naccache D, editor. Proceedings of topics in cryptology - CT-RSA. Lecture notes in computer science, vol. 2020. San Francisco: Springer-Verlag; 2001.

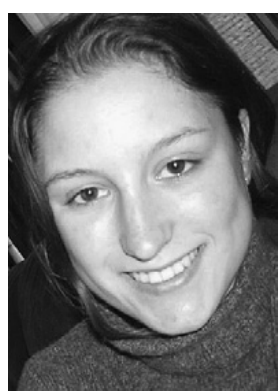

Elke De Mulder was in 1981 in Belgium. From 1999 to 2004 she studied at the "Katholieke Universiteit Leuven" where she got her degree in July 2004 with the thesis "Electromagnetic Analysis (EMA) of a FPGA implementation of an elliptic curve cryptosystem". Currently she is a Ph.D. student at COSIC. The topic of her research is countermeasures against electromagnetic analysis (EMA). Her research is funded by the IWT-Flanders.

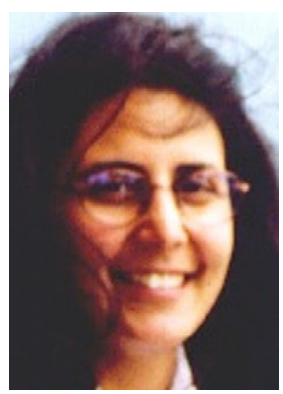

S. Berna Örs received the Electronics and Communication Engineering Degree in 1995 and the M.Sc. Degree in Electronics and Communication Engineering in 1995 from the Istanbul Technical University, in Istanbul, Turkey. She received the Ph.D. Degree in applied sciences from the Katholieke Universiteit Leuven, in Leuven, Belgium in 2005. She is currently assistant professor at Istanbul Technical University. Her interests include circuits, processor architectures and embedded systems in application domains such as security, cryptography, digital signal processing and wireless applications.

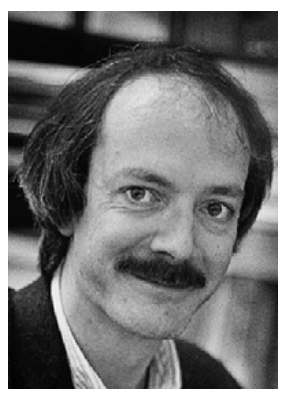

Bart Preneel received the Electrical Engineering degree and the Doctorate in Applied Sciences from the Katholieke Universiteit Leuven (Belgium). He is currently professor (hoogleraar) at the Katholieke Universiteit Leuven and visiting professor at the T.U. Graz in Austria. He was visiting professor at several universities in Europe (Ghent, Belgium, Bergen, Norway and Bochum, Germany). During the academic year 1993-1994, he was a research fellow of the EECS Department of the University of California at Berkeley. His main research interests are cryptography, network security, and wireless communications. He has authored and co-authored more than 180 scientific publications and is an inventor of two patents. He is vice president of the International Association for Cryptologic Research (IACR) and a member of the Editorial Board of the Journal of Cryptology, the IEEE Transactions on Informations and Security, and the ACM Transactions on Information Security. He is also a Member of the Accreditation Board of the Computer and Communications Security Reviews (ANBAR, UK). He has participated to more than 15 research projects sponsored by the European Commission, for four of these as project manager. He is currently project manager of the European Network of Excellene ECRYPT. In 2003, he has received the European Information Security Award in the area of academic research, and he received an honorary Certified Information Security Manager (CISM) designation by the Information Systems Audit and Control Association (ISACA). Since 1989, he is a Belgian expert in working group ISO/IEC JTC1/SC27/WG2 (Security Techniques and Mechanisms), where he has edited five international standards. 


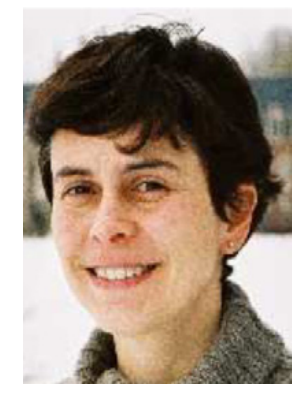

Ingrid Verbauwhede received the Electrical Engineering Degree in 1984 and the Ph.D. Degree in applied sciences from the K.U. Leuven, in Leuven, Belgium in 1991. She was a lecturer and visiting research engineer at UC Berkeley from 1992 to 1994 . From 1994 to 1998 she was a principal engineer first with TCSI and then with Atmel in Berkeley, CA. She joined UCLA in 1998 as an associate professor and the K.U. Leuven in 2003. Her interests include circuits, processor architectures and design methodologies for realtime, embedded systems in application domains such as security, cryptography, digital signal processing and wireless applications. Prof. Verbauwhede was the general chair of the IEEE International Symposium on Low Power Electronic Devices (ISLPED) in 2003. She is or was a member of several program committees, including DAC, ISSCC, DATE, CHES, ICASSP, SIPS, ASAP. She is the design community chair on the 42nd and 43th DAC executive community. She is a senior member of IEEE. 